\title{
Motorized Spiral Enteroscopy: to infinity and beyond?
}

\author{
T.G. Moreels, L. Monino \\ Department of Gastroenterology \& Hepatology, Cliniques universitaires Saint-Luc, Brussels, Belgium.
}

\section{To the Editor,}

With the advent of device-assisted enteroscopy (DAE) in the early 2000s, endoscopic access to the entire small bowel is possible nowadays (1). And yet, there is still room for improvement. Total enteroscopy remains a time-consuming procedure, often combining the antegrade (oral) and retrograde (anal) approach with only a reasonable chance to obtain complete endoscopy of the entire small bowel (2). Therefore, the aim is to go faster, deeper and to perform more advanced therapeutic interventions within the long and tortuous small bowel. Moreover, DAE was also shown to be effective to perform endoscopic retrograde cholangiopancreatography (ERCP) in patients with surgically altered anatomy and to complete colonoscopy in patients with previously incomplete conventional colonoscopy due to long dolichocolon (3).

The latest DAE development is Motorized Spiral Enteroscopy (MSE), initially conceptualized as a manually driven rotational spiral overtube by Paul A. Akerman, and further developed and commercialized as a motorized spiral overtube by the Olympus Medical Systems Corporation (4). Initial feasibility trials have shown that MSE can compete with already available DAE techniques (single- and double-balloon enteroscopy) with regard to diagnostic yield and endotherapy within the small bowel $(5,6)$. However, being a short type enteroscope of $168 \mathrm{~cm}$ (as compared to the $200 \mathrm{~cm}$ long single- and double-balloon enteroscopes), MSE appears to be even more effective in obtaining deep and total enteroscopy with a relatively short procedural duration $(2,6)$. In addition, the working channel diameter is increased to $3.2 \mathrm{~mm}$ (as compared to $2.8 \mathrm{~mm}$ ) with an extra irrigation channel, facilitating therapeutic interventions within the small bowel. This faster and deeper (but more aggressive) enteroscopy technique comes with the price of an increased risk of mucosal injuries (ranging from superficial bruising to laceration and even perforation) within the oesophagus and the small bowel, luckily remaining asymptomatic most of the time without any clinical consequence (6). So far, this promising new technique has the potential of becoming a gamechanger in the still evolving field of deep enteroscopy.

Moreover, apart from the endoscopic exploration of the small bowel, early reports are emerging showing that MSE might also be a useful tool for indications beyond the small bowel, like colonoscopy and ERCP.
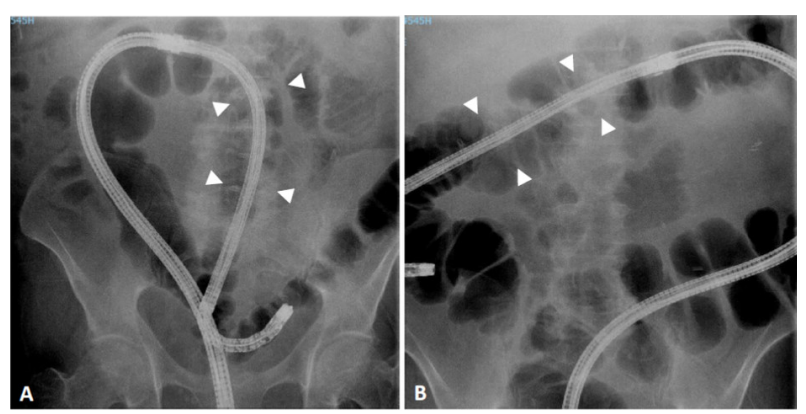

Figure 1. - Fluoroscopic image of the introduction of the PSF1 motorized spiral enteroscope in a 51-year old male patient with a long dolichocolon with previously failed conventional colonoscopy and even double-balloon enteroscopy-assisted colonoscopy (A). The colon was effectively shortened and the caecum was reached in 15 min allowing complete colonoscopy (with additional polypectomy in the caecum) (B). Arrows indicate the spiral overtube.

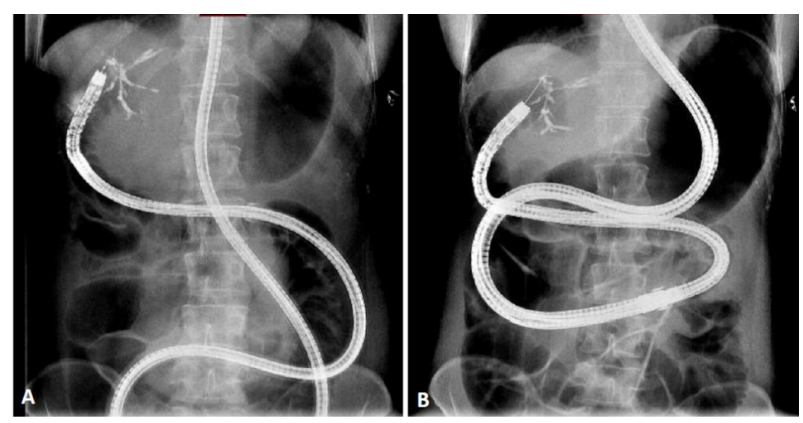

Figure 2. - Fluoroscopic image of a single-balloon enteroscopy-assisted ERCP in a 39-year old female with a history of split liver transplantation with a Roux-en-Y reconstruction (A) and a second procedure using the SPF-1 motorized spiral enteroscope (B). Notice the shorter position of the motorized spiral enteroscope in $\mathrm{B}$ as compared to the single-balloon enteroscope in A.

Although prospective trials are currently not available, Figure 1 illustrates that MSE is effective to complete colonoscopy after failed conventional and even doubleballoon enteroscopy-assisted colonoscopy. And Figure 2 illustrates its feasibility to perform ERCP in a patient with surgically altered anatomy, as compared to singleballoon enteroscopy-assisted ERCP in the same patient.

Correspondence to : Tom G. Moreels, Department of Gastroenterology \& Hepatology, Cliniques universitaires Saint-Luc, Avenue Hippocrate 10, 1200 Brussels, Belgium.

Email: tom.moreels@uclouvain.be

Submission date : 30/07/2021

Acceptance date : $12 / 08 / 2021$ 
These figures currently only represent anecdotal reports of successful MSE procedures beyond the indication of small bowel exploration. They also illustrate the long way ahead of prospective clinical trials imperative to correctly position MSE within the armamentarium of endoscopic techniques to explore the small bowel, and beyond... One of the most important open questions remains whether MSE is able to replace single- and double-balloon enteroscopy, or whether the different DAE techniques are complementary with their own proper and delineated indications. The answer to this burning question is currently unknown. However, it is eagerly awaited since it represents important information for endoscopy units planning to invest in enteroscopy equipment.

Key words: device-assisted enteroscopy, motorized spiral enteroscopy

\section{Conflict of interest:}

T.G. Moreels and L. Monino received speaker's fees from Olympus Belgium and Olympus Europe.

\section{References}

MOREELS T.G. History of endoscopic devices for the exploration of the small bowel. Acta Gastroenterol. Belg., 2009, 72: 335-337.

BEYNA T., ARVANITAKIS M., SCHNEIDER M., GERGES C., HOELLERICH

J., DEVIERE J., et al. Total motorized spiral enteroscopy: first prospective clinical feasibility trial. Gastrointest Endosc., 2021, 93: 1362-1370.

MOREELS T.G. Update in enteroscopy: new devices and new indications. Dig. Endosc. 2018, 30: 174-181.

AKERMAN P.A., AGRAWAL D., CANTERO D., PANGTAY J. Spiral enteroscopy with the new DSB overtube: a novel technique for deep peroral smallbowel intubation. Endoscopy, 2008, 40: 974-978.

BEYNA T., ARVANITAKIS M., SCHNEIDER M., GERGES C., BOING D, DEVIERE J., et al. Motorised spiral enteroscopy: first prospective clinical feasibility study. Gut, 2021, 70: 261-267.

BARKIN J.A., LEIGHTON J.A. Continuation of a fantastic voyage! From push and pull to a new novel motorized spiral enteroscope: the next chapter in deep enteroscopy. Gastrointest. Endosc. 2021, 93: 627-629. 